\title{
Design of Intelligent Controller for Active Magnetic Bearing System based on DSP
}

\author{
Gong jing-wen ${ }^{1,2}$ \\ ${ }^{1}$ School of Mechanical \&Electrical Engineering \\ Wuhan University of Technology \\ Wuhan, China \\ ${ }^{2}$ Department of Control Science and Engineering \\ Huazhong University of Science and Technology \\ Wuhan, China \\ jingwen_gong@163.com \\ Zhang zhu \\ School of Mechanical \&Electrical Engineering \\ Wuhan University of Technology \\ Wuhan, China
}

\author{
Hu ye-fa \\ School of Mechanical \&Electrical Engineering \\ Wuhan University of Technology \\ Wuhan, PR China
}

\begin{abstract}
Based on TMS320C6713 DSP of TI and XC95288XL CPLD of Xilinx, the hardware circuit of the controller is designed for the five-degree-freedom active magnetic bearing(AMB) flexible rotor system. Under the CCS3.3 development environment, the design uses C language to program a position PID control algorithm. The results of experiment show that magnetic bearing control system has strong robustness and anti-jamming capability using this controller.
\end{abstract}

Keywords-active magnetic bearing; TMS320C6713 DSP CPLD; position PID

\section{INTRODUCTION}

Active Magnetic Bearing (AMB) uses controllable electromagnetic force to levitate the shaft and these bearings are mainly composed by rotor, electromagnet, sensor, controller, and a power amplifier ${ }^{[1]}$. As realizing no mechanical contact, also realizing electronic control, active magnetic bearings have the advantages of no mechanical contact and no lubrication, compared with plain bearings. The advantages can avoid mechanical friction and wear between the rotor and the support, extend equipment life, improve equipment operating conditions. The AMB controller systems have broad application prospects in transportation, metallurgy, machinery manufacturing and aerospace ${ }^{[2]}$.

Performance of the controller is one of the key issues to achieve a stable suspension of AMB. Against to improve the performance of AMB, Xiaoyan Xue designed a AMB controller based on TMS320F2407 and did the simulation of Single-degree-of-freedom control system ${ }^{[3]}$. Hong QIU achieved the ball stable suspension based on TMS320F2812 controller ${ }^{[4]}$. Alexander Smirnov designed a magnetic bearing controller based on TMS320F28335, and did LQG control algorithm simulation ${ }^{[5]}$. Taking the requirement of the processing speed for complex control algorithm into account, this paper used TMS320C6713 DSP to design a five-degree-of- freedom active magnetic bearing controller, realized 5 inputs and 10 outputs of real-time signal acquisition and processing, adopted anti-windup position PID control algorithm, and did some experimental studies.

\section{WORKING PRINCIPLE OF AMB}

The electromagnets of AMB control system install on the stators. The rotor suspends in the magnetic field generated by the electromagnets. One or more sensors are installed on each electromagnet to detect shaft position. According to the signal of the sensors, the controller corrects the electromagnet current to control the attraction of the electromagnet. Then the rotor is suspended in a target position. So that the AMB control system achieves a certain accuracy requirements ${ }^{[1]}$. The diagram of system is shown in figure 1.

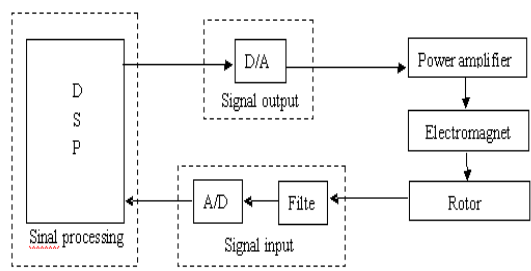

Fig.1. Block diagram of AMB working

\section{DESIGN OF HARDWARE CIRCUIT}

AMB controller consists of power supply module, $\mathrm{A} / \mathrm{D}$ converter module, $\mathrm{D} / \mathrm{A}$ converter module, etc. $\mathrm{A} / \mathrm{D}$ module collects the detection signal of sensors and converts the analog signal to a digital signal. After processing of DSP algorithm, the output digital signal is sent to the $\mathrm{D} / \mathrm{A}$ module. Then $\mathrm{D} / \mathrm{A}$ module converts it to analog signal. The block diagram of the controller is shown in Figure 2. 


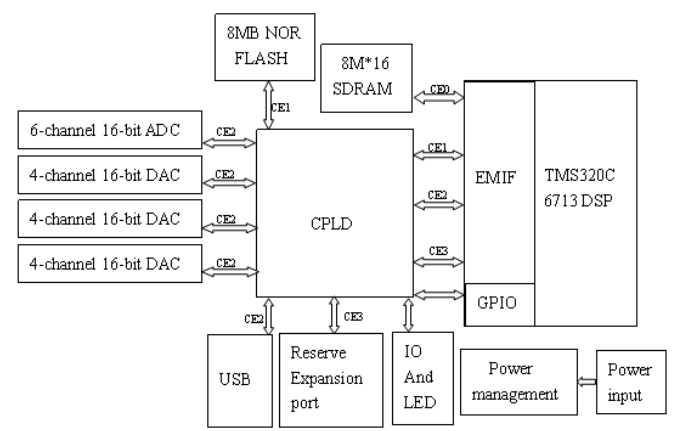

Fig.2. Block diagram of the controller

\section{A. Power of controller}

The design of the power circuit should consider not only the voltage accuracy, stability and peripheral circuit complexity, but also the issue of power. In addition, according to the design process, the power up sequence need to follow a certain order to ensure the normal working of the chip on the system when DSP power up and shutdown. Otherwise, the result will degrade device performance or cause permanent damage. TMS320C6713 DSP core requires $1.2 \mathrm{~V}$ power supply, I/O requires $3.3 \mathrm{~V}$. The power circuit should guarantee that the kernel is powered on earlier than the $\mathrm{I} / \mathrm{O}$ interface or they are powered on at the same time. This paper makes two power chips TPS54310 working at the same time by giving two SS/ENA pins voltage exceeding $1.2 \mathrm{~V}$, in order to ensure the DSP core and I/O interface powered up at the same time. $3.3 \mathrm{~V}$ power supply circuit is shown in Figure 3 below. $1.2 \mathrm{~V}$ power supply circuit is similar with $3.3 \mathrm{~V}$, just need to change some values of resistances.

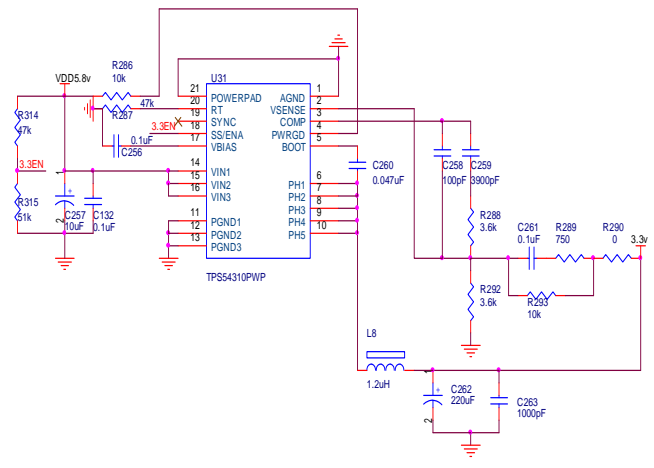

Fig.3. 3.3V power supply circuit

\section{B. Design of $A / D$ conversion}

Since the influence of electromagnetic interference at the measurement field, the sensor signal often contains a variety of frequency components of the noise signal. So the paper designs a RC filter in the front of $A / D$.

In the paper, the $A / D$ converter chooses a 6-channel simultaneous sampling 16-bit analog-to-digital converter ADS8365 of TI. 6 analog channels are divided into three groups, A, B and $\mathrm{C}$. Each has a holding signal (HOLDA, HOLDB and HOLDC) and the holding signal is used to start the $\mathrm{A} / \mathrm{D}$ converter. 6 channel offer synchronous parallel sampling and conversion. A conversion is initiated on the ADS8365 by bringing the HOLDX pin low for a minimum of $20 \mathrm{~ns}$. When the converting results are sent to the output register, the EOC pin will outputs half a clock cycle of low level and prompts DSP to accept the conversion results.

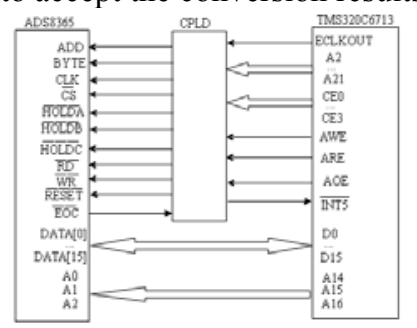

Fig.4. A/D conversion interface diagram

In this design, $\mathrm{A} / \mathrm{D}$ conversion uses a single-ended inputting mode to gather an analog signal of $0 \sim 5 \mathrm{~V}$. -IN inputs the common mode voltage of $2.5 \mathrm{~V}$. + IN swings around $2.5 \mathrm{~V}$. The peak-to-peak amplitude of the differential voltage are $2.5 \mathrm{~V}+\mathrm{V}_{\mathrm{REF}}$ and $2.5 \mathrm{~V}-\mathrm{V}_{\mathrm{REF}}$. ADC hardware is controlled by CPLD. DSP only needs to seed a conversion command to CPLD. Then, conversion begins. The logic control diagram is shown in Figure 4.

\section{D/A conversion realize}

The digital signal is converted to an analog signal by $\mathrm{D} / \mathrm{A}$ conversion. Then the analog signal is sent to power amplifier. So the value of the coil current can be controlled. The paper chooses DAC8544 to D/A converter. DAC8544 is a low-power, quad-channel and 16-bit voltage output DAC. Due to controlling a 5-degree-of-freedom AMB needs 10 route circles, the design uses three DAC8544. There are 12 D/A channels in order to meet the quantity requirements of 10 coils. $\mathrm{D} / \mathrm{A}$ conversion circuit diagram is shown in Figure 5.

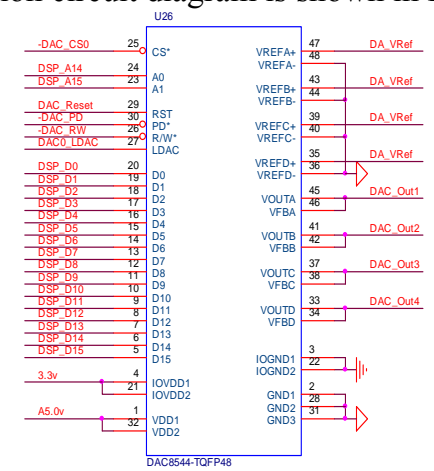

Fig.5. D/A conversion circuit diagram

CPLD finishes address decoding through 3 address lines of A16 A18 for three DAC8544. The work process of DAC8544 is as follows: Firstly, DSP writes the value, which need to be converted, to the channel of the DAC8544 register. Secondly, DSP starts to send the conversion commend, which makes LDAC high. Then, DAC8544 begins to convert. At last, DAC8544 outputs the voltage after converting. The conversion timing diagram is shown in Figure 6. 


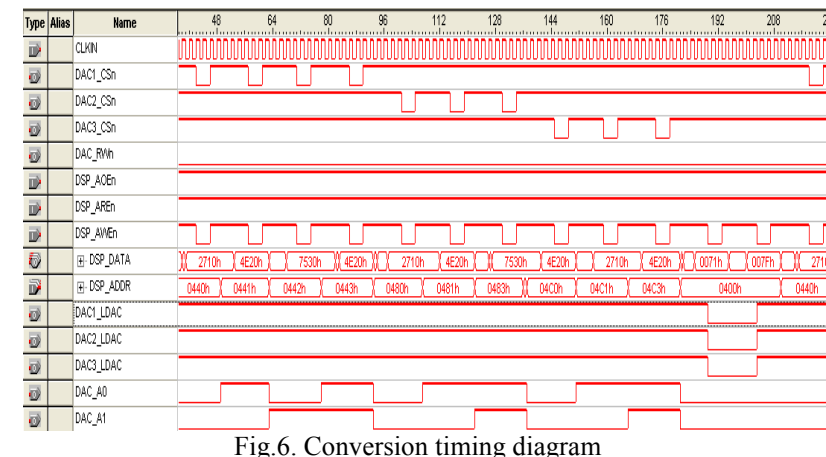

IV. SOFTWARE DESIGN OF CONTROLLER

\section{A. Software structure of controller}

The control system software is written by $\mathrm{C}$ language under CCS3.3 integrated development environment. Software structure is divided into DSP initialization module, A/D acquisition module, PID algorithm processing module and D/A conversion module. The block diagram is shown in Figure 7.

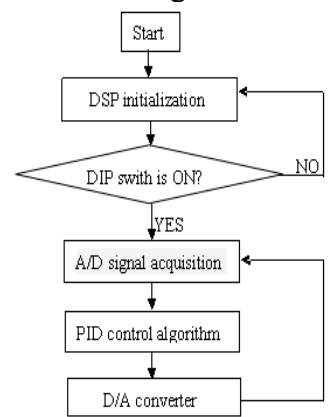

Fig.7. Control system software flowchart

\section{B. PID control algorithm}

In control theory, PID control is one of the most widely used and most mature control technology. PID is easy to tune parameter and has excellent robustness. The control effect of PID is more significant in most industrial control occasions. The parameters of Conventional PID controller are often poor tuning and the performance is poor, also. So, in a variety of industrial control, can not only use the conventional PID control, but also use the modified form of the various PID based on the requirements of the system. For example, PID control with dead zone, integral separation PID control, incomplete derivative PID control and so on. The control algorithm is anti-windup position type PID in this paper. Anti-windup PID block diagram is shown in Figure 8.

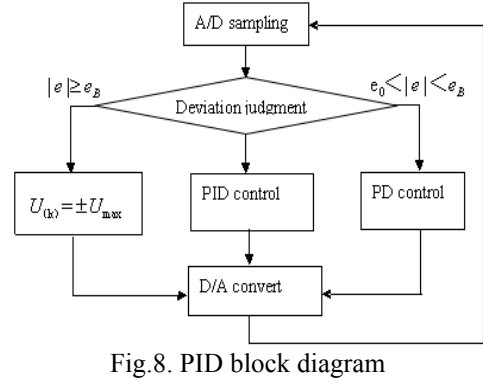

\section{AMB SUSPENSION TEST}

In magnetic levitation control system, real-time control of controller's signal is one of an important performance factors. Real-time control relates to the level of dynamic response capability and the size of stiffness. For the rotor of $60000 \mathrm{r} / \mathrm{min}$, rotor is controlled 4 times per revolution, then the control period is $250 \mu \mathrm{s}$. Before the suspension test, the executed time of $\mathrm{A} / \mathrm{D}, \mathrm{D} / \mathrm{A}$, and PID algorithm should be tested. The executed time is $29.94 \mu$ s which is much smaller than the requirement of system $250 \mu$ s.

This test makes magnetic bearing to be suspended stably by anti-integral saturation position PID based on TMS320C6713 DSP. The experiment makes rotor whirling by adjusting the power frequency to $150 \mathrm{~Hz}$. After the rotor whirl, PCI1711 capture card collects the value of sensor and sends the value to host computer. Then, the values are displayed by MATLAB. Rotary effect diagram is shown in Figure 9.

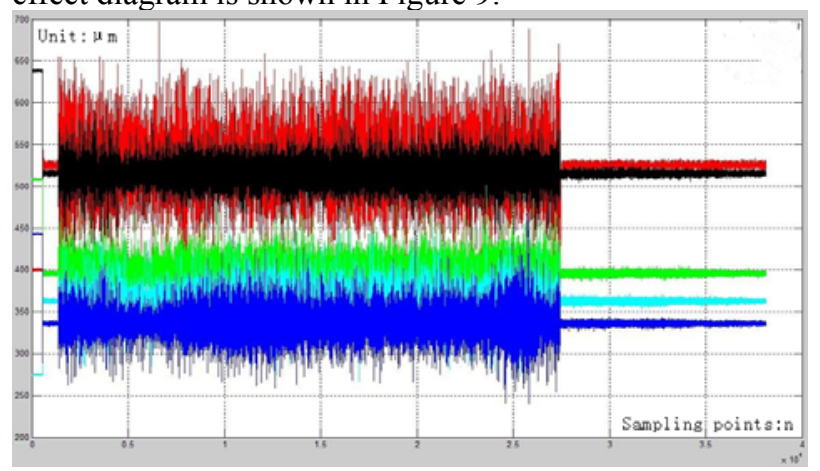

Fig.9. Rotary effect diagram

\section{CONCLUSION}

This paper designs a magnetic bearing controller based on TMS320C6713 DSP to realize the stable suspension of magnetic bearing. The result of maglev bearing suspension experimental shows that the control accuracy achieves a certain effect. The controller is able to meet the real-time requirement of magnetic bearing's stable suspension. The next plane is to improve rotate speed, optimize the design of the controller and use a different control algorithm to realize the stable suspension of the $60000 \mathrm{r} / \mathrm{min}$ speed requirements. 


\section{ACKNOWLEDGMENT}

This research is supported by the Natural Science Foundation of China (No. 51175390, No. 51205300).

\section{REFERENCES}

[1] Yefa Hu, Zude Zhou, Zhengfeng Jiang. The basic theory and application of magnetic bearings[M].Machinery Industry Press, 2006.

[2] Hongwei Jiang, Zhenyu Xie. The maglev system digital controller based on TMS320F 28335 DSP[J]. Mechanical and electronic, 2010(11):29-32.

[3] Xiaoyan Xue, Zhifeng Gu, Wensheng Liu, Jie Li. Active magnetic bearing digital controller based on DSP and CPLD $[\mathrm{J}]$. Electrical switches, 2007(5):27:29.

[4] Hong QIU, Guangzhong CAO, J.F.PAN, Lv-ming LIN. The Development of Magnetic Levitation Ball Control System Based on TMS320F2812[J]. IEEE,2009,1:1-4.

[5] Alexander Smirnov, Kimmo Tolsa, Rafal P.Jastrzebski Implementation of a bumpless switch in axial magnetic bearings [J]. IEEE, 2010:63-68 